\title{
Satisfacción laboral del personal en el Servicio de Nefrología del Hospital Guillermo Kaelin de la Fuente (Villa María) en 2019
}

\author{
Yojani Holguin Morales ${ }^{1, a}$; Carlos Contreras Camarena* b \\ RESUMEN
}

Objetivo: Identificar el nivel de satisfacción laboral del personal del Servicio de Nefrología del Hospital Guillermo Kaelin de la Fuente (Villa María) en 2019.

Materiales y métodos: Estudio de tipo analítico. El Servicio de Nefrología cuenta con 76 trabajadores, 40 (52,64 \%) participaron en el estudio (entre médicos, licenciados en enfermería, técnicos en enfermería, administrativos, etc.) Se evaluaron las dimensiones extrínseca e intrínseca de la satisfacción laboral. Los datos se procesaron con el software SPSS, versión 24.00 .

Resultados: La población laboral fue relativamente joven (edad media de 38,8 años y DE: $\pm 6,8$ ), con discreta predominancia del sexo femenino (67,50\%). El $28 \%$ de los trabajadores tuvo un nivel alto de satisfacción. Los participantes que iniciaron sus labores desde la fundación del hospital (tiempo de servicio $\leq 3$ años) tuvieron mayor satisfacción laboral que el personal de salud con tiempo de servicio entre 4 y 6 años. El 86,7 \% de los trabajadores con menos de 3 años de servicio mostraron un mayor grado de satisfacción media-alta, comparado con el 68 \% obtenido en el grupo que laboraba entre 4 a 6 años.

Conclusiones: El $28 \%$ de los trabajadores tuvieron nivel alto de satisfacción laboral, de ellos, los médicos obtuvieron el mayor grado de satisfacción (83,3\%). El 48 \% tuvo un nivel medio de satisfacción; además, uno de cada cuatro trabajadores mostró un bajo nivel de satisfacción. Los trabajadores con tiempo de servicio $\leq 3$ años fueron los que mostraron el mayor nivel de satisfacción laboral. Observamos que un menor tiempo de servicio se obtiene un mayor nivel de satisfacción laboral.

Palabras clave: Satisfacción laboral; Personal de salud; Ambiente de trabajo (Fuente: DeCS BIREME)

\section{Job satisfaction of the nephrology department staff of the Hospital Guillermo Kaelin de la Fuente, Villa María, 2019}

\section{ABSTRACT}

Objective: To identify the level of job satisfaction of the nephrology department staff of the Hospital Guillermo Kaelin de la Fuente, Villa María, in 2019.

Materials and methods: An analytical study was conducted. The nephrology department consists of 76 staff members, out of which $40(52.64 \%)$ took part in the study (physicians, registered nurses, nurse technicians, administrative staff, etc.). The extrinsic and intrinsic dimensions of job satisfaction were evaluated. The data was processed using SPSS Statistics statistical software, version 24.00 .

Results: The staff members were relatively young (mean age: 38.8 years; SD: \pm 6.8 ) and females slightly prevailed (67.50\%). Twenty-eight percent ( $28 \%$ ) of the staff members had high levels of satisfaction. The study participants who started working in the hospital from its creation (job tenure $\leq 3$ years) showed higher levels of job satisfaction than those with a job tenure between 4 and 6 years. Eighty-six point seven percent (86.7\%) of staff members who worked less than 3 years showed higher levels of medium-high satisfaction than those who worked between 4 and 6 years (68\%).

Conclusions: Twenty-eight percent ( $28 \%)$ of the staff members had a high level of job satisfaction, out of whom physicians showed the highest level of satisfaction (83.3\%). Forty-eight percent (48\%) had a medium level of job satisfaction and one out of four staff members showed a low level of satisfaction. Staff members with a job tenure $\leq 3$ years had the highest level of job satisfaction: the shorter the job tenure, the greater the job satisfaction. Apparently, job satisfaction "fades away" as years pass.

Keywords: Job satisfaction; Health personnel; Working environment (Source: MeSH NLM).

1 Hospital Guillermo Kaelin de la Fuente, Servicio de Nefrología. Lima Perú.

a Enfermera especialista en Nefrología.

b Médico internista, Magister en Gerencia de Servicios Públicos de Salud.

* Autor corresponsal 


\section{INTRODUCCIÓN}

La satisfacción laboral se define como la sensación de bienestar del trabajador al desempeñar su labor. Ha sido abordada y definida por diversas corrientes científicas y metodológicas, pero hasta la fecha no existe consenso respecto a la definición de satisfacción laboral, a pesar de estar relacionada a múltiples variables intrínsecas como eficiencia, desempeño laboral y comportamiento organizacional. Así, Wright y Bonett plantean que "La satisfacción laboral es probablemente la más común y más antigua forma de operacionalización de la felicidad en el lugar de trabajo" (1). Shaun la define como "el conjunto de sentimientos favorables que expresan los miembros de una organización con respecto a la visión, misión, objetivos y procesos de la institución, así como el orgullo que sienten los empleados por pertenecer a la organización" (2). Es frecuente observar diversas tipos de interacción entre trabajadores y empleadores y/o directivos. Cada una de estas personas tiene diferentes formas y estándares de comportamiento relacionados a sus roles y circunstancias específicas. La suma de todos estos comportamientos, sus causas y motivos, sus interrelaciones y su compromiso es denominado comportamiento organizacional (3). De acuerdo con Schermerhorn (2005), "la satisfacción laboral determina la decisión de pertenecer a una organización y permanecer como miembro de la misma, y la decisión de desempeño", es decir, trabajar con el mayor esfuerzo y así lograr una mejor productividad y altos niveles de desempeño en su centro de labores ${ }^{(4)}$.

Algunos autores expresan definiciones mucho más homogéneas respecto a la satisfacción y la definen como la expresión intrínseca - extrínseca de una variable multidimensional. Munchinsky describe claramente estos dos componentes fundamentales: los factores extrínsecos están relacionados al entorno del trabajo y los factores intrínsecos reflejan la experiencia de las personas con su acción laboral. La satisfacción extrínseca abarca factores como el salario adecuado y justo, las políticas políticas de la compañía, los recursos, la supervisión, la interacción con los compañeros de trabajo, las oportunidades de promoción, la relación con los usuarios o clientes y la seguridad en el trabajo. La satisfacción intrínseca está asociada al reconocimiento del trabajador de su labor, responsabilidad, promoción y aspectos relativos al trabajo mismo; es decir, la satisfacción intrínseca es el resultado de las recompensas de índole interna al propio trabajador ${ }^{(5)}$.

Falatah et al. sugieren que los diseños de trabajo reconfigurados para construir y cultivar vínculos entre las diferentes disciplinas en salud serán necesarios para mejorar la satisfacción laboral y los niveles de compromiso de los trabajadores. Esto se puede lograr mediante reuniones de equipos multidisciplinarios frecuentes y oportunas que fomenten un intercambio de conocimientos precisos y específicos (mejora del conocimiento mutuo), una mejor alineación de objetivos, menos pérdidas de procesos y una resolución de problemas más efectiva entre equipos interdisciplinarios. Estas actividades servirán para influir positivamente los resultados relacionados (6). El objetivo de este estudio fue determinar el nivel de satisfacción laboral del personal que trabaja en el Servicio de Nefrología del Hospital Guillermo Kaelin de la Fuente del distrito de Villa María (Lima, Perú) en 2019.

\section{MATERIALES Y MÉTODOS}

\section{Diseño y población de estudio}

Estudio de tipo cuantitativo, analítico y de corte transversal. La población estudiada fueron los trabajadores del Servicio de Nefrología registrados en la planilla oficial del hospital (según datos proporcionados por la Oficina de Personal del Hospital Guillermo Kaelin de la Fuente). Se incluyeron 40 participantes de un universo de 76 trabajadores asistenciales entre médicos, licenciados en enfermería, técnicos en enfermería y empleados administrativos. Se excluyeron de la población total a los trabajadores con menos de tres meses de permanencia en la institución, los trabajadores ausentes en el momento de la aplicación del instrumento (comisión, vacaciones, destacados, etcétera) y trabajadores con problemas psicológicos graves o psiquiátricos. La información se ingresó a la base de datos SPSS versión 24.

\section{Variables y mediciones}

Cuarenta trabajadores fueron incluidos en la investigación, a todos ellos se les aplicó un cuestionario sobre Satisfacción Laboral creado el 2014 por Montoya ${ }^{(7)} y$ modificado por Neyra y Salinas ${ }^{\left({ }^{8}\right)}$ en el 2017. Para efectos de esta investigación el cuestionario se sometió a juicio de expertos para determinar la validez del contenido, de constructo y de criterio, y luego se realizó una prueba piloto para probar el grado de comprensión por los participantes. El instrumento fue estructurado en 2 partes: la primera destinada a la recolección de datos generales y socioeconómicos, la segunda para recoger los datos específicos de satisfacción laboral en sus dos dimensiones: extrínseca (que comprende el sueldo, supervisión, comunicación, beneficios laborales, compañeros de trabajo, condiciones ambientales y físicas) e intrínsecas (que abarca el trabajo en sí, recompensas, reconocimiento y promoción). Para identificar las características de la satisfacción laboral en el personal de salud se empleó un cuestionario con 40 ítems (que utiliza la escala de Likert) donde el puntaje más bajo fue 1 (Totalmente en desacuerdo) y el puntaje más alto fue 5 (Totalmente de acuerdo). 


\section{Análisis estadístico}

Los datos recolectados fueron codificados, ingresados en una base de datos y procesados con el paquete estadístico SPSS 24. Se aplicaron estadísticos de tendencia central y de dispersión, chi cuadrado para análisis de las variables cualitativas y coeficiente de correlación de Pearson para determinar la correspondencia entre grupos ocupacionales y satisfacción laboral.

\section{Consideraciones éticas}

En todo momento de la investigación se mantuvo la confidencialidad pertinente de la información obtenida de la base de datos proporcionada por la Oficina de Recursos Humanos de la institución.

\section{RESULTADOS}

La edad promedio de la población fue 38,8 años (DE: $\pm 6,8$ ) con predominio del grupo etario entre 30 y 39 años (60\%). El sexo predominante fue el femenino $(67,50 \%)$. El 52,50\% de la población encuestada fueron personal no profesional (técnico auxiliar y administrativo). La mayoría de los participantes laboraban en el servicio entre 4 a 6 años (62,50\%), los trabajadores con tiempo de servicio de 6 años estuvieron trabajando desde la inauguración del hospital. De los encuestados, 39 estaban en el grupo de personal permanente. El $72 \%$ de los trabajadores percibía salarios entre 1000 a 3000 soles y solo el $15 \%$ tenía como salario mensual más de 5000 soles (Tabla 1).

Tabla 1. Variables generales y socioeconómicas de la población de estudio (n: 40). Hospital Guillermo Kaelin de la Fuente (Lima, Perú)

\begin{tabular}{|c|c|c|c|}
\hline Variable & Descripción & Cantidad & $\begin{array}{c}\text { Porcentaje } \\
\text { (\%) }\end{array}$ \\
\hline \multirow[t]{2}{*}{ Sexo } & Masculino & 13 & 32,50 \\
\hline & Femenino & 27 & 67,50 \\
\hline \multirow{4}{*}{$\begin{array}{l}\text { Edad } \\
\text { (años) }\end{array}$} & $20-29$ & 1 & 2,50 \\
\hline & $30-39$ & 24 & 60,00 \\
\hline & $40-49$ & 12 & 30,00 \\
\hline & 50 a más & 3 & 7,50 \\
\hline \multirow{3}{*}{ Estado civil } & Soltero (a) & 20 & 50,00 \\
\hline & Casado (a) & 13 & 32,50 \\
\hline & Conviviente & 7 & 17,50 \\
\hline \multirow{4}{*}{ Ocupación } & Médico & 6 & 15,00 \\
\hline & Enfermera & 13 & 32,50 \\
\hline & Técnico en enfermería & 16 & 40,00 \\
\hline & Otros & 5 & 12,50 \\
\hline \multirow{3}{*}{$\begin{array}{c}\text { Tiempo de servicio } \\
\text { (años) }\end{array}$} & Menos de uno & 5 & 12,50 \\
\hline & 1 a 3 & 10 & 25,00 \\
\hline & 4 a 6 & 25 & 62,50 \\
\hline \multirow[t]{2}{*}{ Estatus laboral } & Temporal & 1 & 2,50 \\
\hline & Permanente & 39 & 97,50 \\
\hline \multirow{3}{*}{$\begin{array}{l}\text { Sueldo promedio } \\
\text { (soles) }\end{array}$} & 1000 a 3000 & 29 & 72,50 \\
\hline & 3000 a 5000 & 5 & 12,50 \\
\hline & 5000 a más & 6 & 15,00 \\
\hline
\end{tabular}


La sumatoria de todas las respuestas reportó una media de 81,15 con una desviación estándar de $\pm 21,64$ y mediante la técnica estadística de estaninos (que cataloga los puntajes de las variables en valor bajo, valor medio y valor alto) se clasificó como nivel bajo de satisfacción laboral al personal de salud que presentó un puntaje entre 40 a 106, nivel medio de satisfacción a los que presentaron un puntaje de 107 a 151 y nivel alto de satisfacción laboral a los que alcanzaron un puntaje entre 152 a 200.

En cuanto a la satisfacción por el tiempo de servicio, se encontró que la mayoría de los trabajadores que estuvieron laborando durante los primeros 3 años tuvieron un mayor nivel de satisfacción laboral óptima que los trabajadores con tiempo de servicio entre 4 a 6 años. Aparentemente, a menor tiempo de servicio es mayor la satisfacción laboral. Al parecer, la satisfacción se va "diluyendo" a medida que pasan los años. El 86,70\% de los trabajadores que laboraban menos de 3 años tuvieron satisfacción aceptable, comparados con el $68 \%$ en los trabajadores que laboraban de 4 a 6 años (se considera como satisfacción aceptable un puntaje entre 107 y 200 puntos) (Tabla 2$)$.

Tabla 2. Satisfacción laboral en el Servicio de Nefrología, según tiempo de servicio. Hospital Guillermo Kaelin de la Fuente

\begin{tabular}{|c|c|c|c|c|c|c|}
\hline & & \multicolumn{5}{|c|}{ Satisfacción laboral } \\
\hline & & & Bajo & Medio & Alto & Total \\
\hline \multirow{4}{*}{$\begin{array}{c}\text { Tiempo de } \\
\text { servicio }\end{array}$} & Hasta 3 & Frecuencia & 2 & 9 & 4 & 15 \\
\hline & años & $\%$ & 13,30 & 60,00 & 26,70 & 100,00 \\
\hline & De $4 \mathrm{a}$ & Frecuencia & 8 & 10 & 7 & 25 \\
\hline & 6 años & $\%$ & 32,00 & 40,00 & 28,00 & 100,00 \\
\hline \multirow{2}{*}{ Total } & & Frecuencia & 10 & 19 & 11 & 40 \\
\hline & & & 25,00 & 47,50 & 27,50 & \\
\hline
\end{tabular}

En el análisis dimensional, la mayoría de los trabajadores tuvieron satisfacción aceptable (grado de satisfacción entre media a alta), $76 \%$ en la dimensión extrínseca y $73 \%$ en la intrínseca. Entre el 25 a $28 \%$ del personal de salud tuvieron un nivel bajo de satisfacción cuando se realizaron el análisis dimensional por separado. Alrededor de un cuarto del total del personal de salud (10 y 11 trabajadores) tuvieron bajo nivel de satisfacción laboral en las dimensiones extrínseca e intrínseca, respectivamente
(Figura 1). En el análisis global se encontró que el nivel de satisfacción medio se acerca al $50 \%$, pero es relevante que alrededor del $25 \%$ de los trabajadores de salud, tienen un bajo nivel de satisfacción laboral y solo aproximadamente un tercio tuvieron un nivel alto de satisfacción. Los resultados por dimensiones separadas fueron muy similares, no se encontró diferencias significativas en el análisis dimensional por separado $(p=0,99)$.

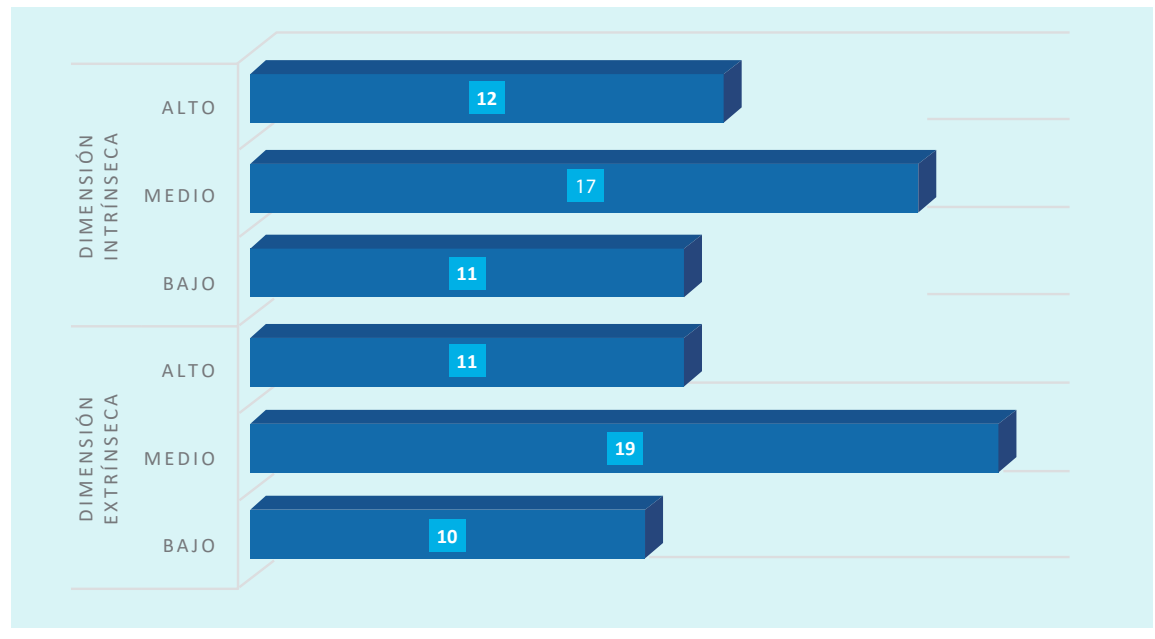

Figura 1. Satisfacción laboral en personal de salud, según dimensiones intrínseca y extrínseca. Hospital Guillermo Kaelin de la Fuente ( $\mathrm{n}=40$ ) 
Se encontraron diferencias significativas en satisfacción laboral entre los grupos ocupacionales estudiados. Los médicos tuvieron el nivel más alto de satisfacción laboral con el $83,3 \%(5 / 6)$, comparado con el $38,5 \%$ encontrado en las enfermeras $(5 / 13)$, los técnicos en enfermería mostraron un $0,00 \%(0 / 16)$, y el grupo de otros, $20 \%$ $(1 / 5)$ (en el grupo de otros se incluyeron a diferentes profesionales como asistenta social, nutricionista, psicóloga y trabajadores administrativos). Las diferencias encontradas en el análisis por grupos ocupacionales fueron estadísticamente significativas $(X 2=17,326$ y $p=0,008)$. Estas diferencias, probablemente, están relacionadas a salarios heterogéneos y a la presencia de algunos beneficios económicos diferenciados dentro del equipo de salud; no existe duda que el grupo ocupacional privilegiado son los médicos, quienes tienen salarios relativamente superiores comparados con los otros trabajadores de salud. En el grupo de licenciadas en enfermería predominó la satisfacción laboral media (puntaje: 107-151), mientras que el nivel alto de satisfacción se presentó en el 38,5\% (5/13). Observamos diferencias significativas entre las enfermeras y los técnicos auxiliares respecto a satisfacción laboral baja o nivel de insatisfacción ( $15,4 \%$ vs. $37,5 \%)$, los técnicos en enfermería tuvieron proporcionalmente 2 veces más de insatisfacción laboral que las enfermeras. No se encontró ningún médico con satisfacción laboral baja, por el contrario, el 83,3 \% de ellos presentaron altos niveles de satisfacción (Figura 2).

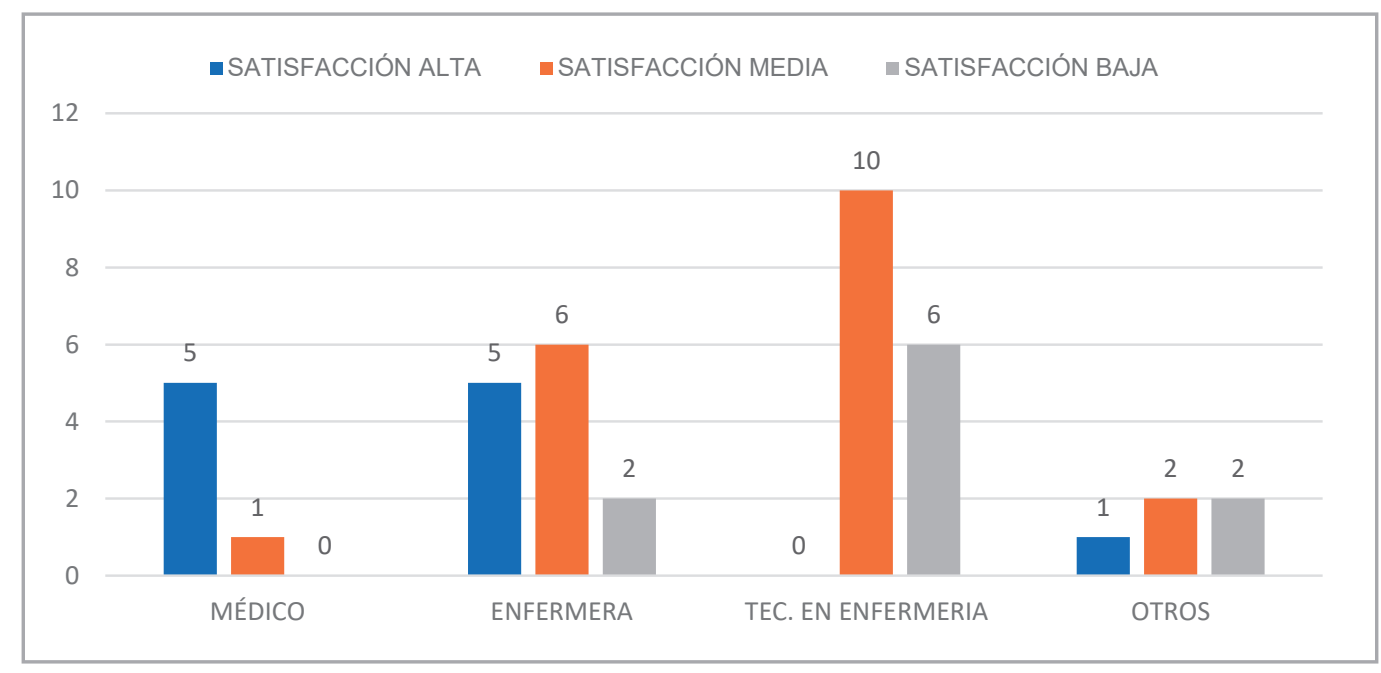

Figura 2. Nivel de satisfacción laboral según, grupo ocupacional $(n=40)$

\section{DISCUSIÓN}

La población que trabaja en el hospital es relativamente joven $(38,8$ años, $D E: \pm 6,8)$, con predominancia del sexo femenino: la relación mujer/ hombre fue 2,07 y difiere de la población de otros estudios en los que la media de edad es relativamente mayor. Estos hallazgos estarían relacionados a políticas gerenciales de recursos humanos de la empresa prestadora de salud ya que estas estas entidades públicoprivadas prefieren convocar a trabajadores relativamente jóvenes. Por otro lado, al ser un hospital de apenas 6 años de fundación, los profesionales de salud probablemente, se localizan en grupos etarios relativamente jóvenes que son potencialmente maleables y contribuyen al alcance de las metas de la institución.

Debido a que uno de cuatro trabajadores muestra un bajo nivel de satisfacción (insatisfecho), es importante que la administración hospitalaria invierta en mejorar la satisfacción de sus trabajadores debido a la multidimensionalidad de esta variable para una evaluación optima ${ }^{(7,8)}$. Pérez Ciordia establece que la satisfacción laboral está vinculada a un grupo de variables como formación continua, equidad en los premios, políticas adecuadas de promoción, relaciones interpersonales y otras. Es decir, para tener trabajadores eficientes es importante contar con un personal capacitado, debidamente entrenado y con beneficios en proporcionalidad a sus logros ${ }^{(9)}$.

Ennuestra investigación, realizada en el Hospital Guillermo Kaelin de la Fuente de la Fuente, Villa María (Lima), el 48 $\%$ de la población total tuvo un nivel medio de satisfacción laboral. Este resultado es discretamente mayor al estudio de Carballo et al. (México) que encontró que el $42 \%$ de los trabajadores de salud estuvo parcialmente satisfecho (en relación a las condiciones de trabajo, infraestructura y equipamiento) y resalta la insuficiencia de los insumos para la ejecución y optimización de los procesos ${ }^{\left({ }^{(10)} \text {. Los }\right.}$ mismos autores reportan una relación directa entre clima laboral institucional y grado de satisfacción y describen que el $76 \%$ de los trabajadores manifestaron que existía un 
clima organizacional favorable al responder positivamente a las preguntas "El ambiente creado por mis compañeros es el ideal para desempeñar mis funciones", "Existe afinidad con mi grupo de trabajo", etc. Indudablemente, las demás variables intervinientes afectan la satisfacción, a pesar de tener un clima organizacional favorable. En su investigación, Salinas et al. enfatiza la estrecha relación entre satisfacción laboral y el desempeño que se vinculan en una relación causa - efecto positiva entre el clima organizacional y las actitudes favorables y desfavorables del trabajador ${ }^{(11)}$.

En nuestra serie el ítem que tuvo mayor valor, dentro del rubro "Trabajo", fue la afirmación "Disfruto de cada labor que realizo en mi trabajo". Es probable que este hallazgo esté relacionado al alto nivel de vocación e identificación de los trabajadores, más que a una satisfacción laboral inherente. Saturno et al. define la satisfacción laboral en las instituciones de salud como un elemento que forma parte de la calidad de atención brindada y aunque se cuestione su relación directa sobre la calidad de atención al cliente externo, no se puede discutir que, al medir la satisfacción laboral, se está comprobando la calidad de la organización y de los servicios internos ${ }^{(12)}$.

La investigación de Carrillo et al. en las Unidades Móviles de Emergencias del Hospital de Murcia (España) analizó la satisfacción laboral de los profesionales de enfermería del centro quirúrgico y utilizó como instrumento la escala de NPT 394 Satisfacción laboral: escala general de satisfacción. La participación fue del $89,65 \%$ y los resultados muestran una satisfacción general media de 58,76\% (DE $\pm 16,64)$, respecto a la investigación extrínseca la media fue de 31,42 $(\mathrm{DE} \pm 9,04)$, y en relación a la intrínseca se obtuvo la media de 27,34 ( $D E \pm 8,22$ ). El factor con el que los profesionales están más satisfechos fue "La estabilidad en el empleo" y con el que estaban menos satisfechos fue "El modo en que se está gestionando" (13).

Los resultados obtenidos tienen una distribución similar a la de nuestra investigación, en que la satisfacción global media no supera el $50 \%$ y la satisfacción en las dimensiones extrínseca e intrínseca alcanzan el 48 y $43 \%$, respectivamente y donde los ítems más representativos y relacionados a satisfacción laboral fueron "Siento que me pagan una suma justa por el trabajo que hago", "Mi sueldo es suficiente para suplir mis necesidades económicas", "Recibo un bono extra por trabajar en un área critica" y "Recibo alimentación por laborar en un área de alto riesgo". Varios autores recomiendan la implementación de medidas destinadas a intervenir los factores que generan la insatisfacción de los profesionales de la salud. A pesar de la aplicación de un instrumento diferente, los resultados de Carrillo et al. se correlacionan perfectamente con los nuestros.

La satisfacción laboral en los servicios de salud está directamente relacionada con la calidad asistencial, de ahí la importancia de su medición en los trabajadores de las instituciones sanitarias. En nuestra investigación encontramos que los más insatisfechos son los licenciados en enfermería y técnicos auxiliares. En la investigación realizada por Carrillo et al. resaltaron importantes diferencias entre categorías ocupacionales respecto a la satisfacción laboral. Encontraron que los grupos de gestión y el de los médicos residentes fueron los más satisfechos, mientras que los facultativos especialistas del área, profesionales y auxiliares de enfermería reportaron menore niveles de satisfacción (14). En nuestro estudio, el resultado del análisis global muestra que uno de cada cuatro trabajadores tiene nivel bajo de satisfacción, pero esta proporción puede incrementarse en el grupo de los técnicos de enfermería y en los trabajadores administrativos en los que el nivel de insatisfacción alcanza el 30 a $40 \%$, respectivamente. La investigación de Fernández et al. (Madrid) muestra que los trabajadores tenían una satisfacción laboral en el rango medio. La tensión laboral y promoción profesional fueron los ítems peor valorados, por lo que los autores recomiendan que los directivos de la institución activen las medidas correctoras necesarias ${ }^{(14)}$.

Los diferentes estudios nacionales e internacionales refieren que solo una minoría de los trabajadores presentan nivel alto de satisfacción laboral. En nuestra serie encontramos que solo alrededor de un tercio $(28 \%)$ de los trabajadores del Servicio de Nefrología presentan nivel alto de satisfacción. En la investigación de Morales et al. en el Servicio de Enfermería del Centro Quirúrgico del Hospital Nacional Alberto Sabogal, encontraron que la mayor parte de los trabajadores $(77,65 \%)$, mostraron un nivel medio de satisfacción y solo un pequeño porcentaje se encontraron totalmente satisfechos; estos resultados resultan preocupantes debido a las consecuencias negativas en la calidad de atención y en el clima organizacional. Estos resultados estarían relacionados al factor vocacional de los trabajadores en el caso del nivel alto de satisfacción. Mientras que los trabajadores con satisfacción laboral media formarían parte del gran grupo de empleados que trabajarían sin estímulo vocacional. Es decir, solamente trabajarían cumpliendo sus tareas asistenciales rutinarias ${ }^{(15) .}$

En los resultados relacionados al grado de satisfacción con el tiempo de servicio, se observó que los trabajadores que tenían mayor tiempo de servicio ( 4 a 6 años) tenían menor grado de satisfacción laboral. Es probable que este hallazgo se relacione con el redireccionamiento de las políticas de contratación del personal. Este proceso fue realizado por la nueva gestión del hospital luego del primer año de creación de la institución y estableció una drástica disminución de los salarios de los trabajadores posteriormente contratados, en relación a los trabajadores que habían ingresado al inicio de las actividades del hospital. 
La satisfacción laboral plena se asocia a una adecuada atención de calidad y, por consiguiente, a una adecuada satisfacción del cliente o usuario. En un análisis teórico realizado en Ecuador, Parra y Arce hallaron una congruencia implícita entre satisfacción laboral y satisfacción del cliente. Concluyeron que las organizaciones deben estar dispuestas a invertir en los recursos humanos (capacitar al personal, incentivos, promociones, bonificaciones, etc.) y así lograr que se brinden una atención de calidad a los usuarios externos en todos los servicios que ofrecen, esto se reflejará en la rentabilidad de la empresa y en la satisfacción del grupo de interés de la organización (16). El manejo del recurso humano es preponderante en las empresas prestadoras de servicios de salud, por lo que dotar de gran satisfacción al personal debe considerarse como una estrategia de primera línea. Por más políticas, manuales, procedimientos, instructivos de trabajos y protocolos de atención que haya implementados la empresa, si el personal no está altamente motivado y con actitud positiva al momento de realizar las actividades asignadas, trasladará esa sensación de inconformidad al servicio prestado y ocasionará la insatisfacción del cliente externo. Parafraseamos a Christopher Lovelock (2011): “Un trabajador satisfecho transmite una actitud positiva y de compromiso organizacional, que se traduce en usuarios del servicio felices y satisfechos, y que aportan a la rentabilidad de la organización" ${ }^{(17) .}$

Salinas et al. realizaron una investigacion con la finalidad de identificar los factores que determinan la satisfacción laboral del personal. El puntaje máximo alcanzado fue de cinco puntos y el mínimo fue cero, y se aceptó un puntaje de 2,5 como suficiente. El personal médico tuvo el mayor grado de satisfacción $(83,3 \%)$, mientras que ninguno del personal técnico auxiliar tuvo satisfacción plena o nivel alto de satisfacción ${ }^{(18)}$. Estos resultados son similares a nuestra investigación: los profesionales médicos fueron los que presentaron la satisfacción laboral más alta en relación con resto de trabajadores $(83,3 \%)$, probablemente, por el papel de liderazgo que el médico ejerce en el equipo de salud y las mejoras salariales que ha recibido este grupo en los últimos 5 años.

En nuestra investigación, es preocupante la magnitud de insatisfacción de los técnicos en enfermería ( 60 \% del total de insatisfacción laboral) de nuestro estudio; ninguno de ellos alcanzó una satisfacción laboral alta. Las dimensiones que tienen mayor incidencia en la insatisfacción de los técnicos de enfermería son la promoción profesional, los salarios inadecuados y la tensión relacionada al trabajo, estos datos son similares a lo descrito por Rodríguez et al. en Asturias ${ }^{(19)}$, quienes investigaron en un hospital español de segundo nivel. Mientras que, en México, el estudio de Pérez et al. ${ }^{(20)}$ encontró que la insatisfacción en este grupo es más frecuente en los hombres que laboran en los turnos vespertino y nocturno. Estos hallazgos, que comprometen específicamente a estos trabajadores, deberían ser el "target" para que los gerentes, administradores y/o directores puedan iniciar medidas correctivas inmediatas para mejorar el grado de satisfacción laboral y no afectar la productividad y la calidad de la atención.

Concluimos que el $28 \%$ de los trabajadores tuvieron nivel alto de satisfacción laboral y menos del 50 \% alcanzó el nivel medio. Los médicos y los trabajadores con un tiempo de servicio de hasta tres años tuvieron mayores niveles de satisfacción laboral. Recomendamos implementar estrategias sostenibles para mejorar la satisfacción laboral en el personal de salud, debido a que menos de un tercio de trabajadores del servicio alcanzaron niveles altos (óptimos) de satisfacción laboral, especialmente, el grupo de técnicos en enfermería, quienes presentaron niveles elevados de insatisfacción.

Contribuciones de los autores: Yojani Holguín Morales: diseño de la investigación, recolección de datos y análisis estadístico. Carlos W Contreras Camarena: Discusión de resultados, análisis crítico y redacción.

Fuentes de financiamiento: Este artículo ha sido financiado por los autores.

Conflicto de Interés: Los autores declaran no tener ningún conflicto de interés.

\section{REFRENCIAS BIBLIOGRÁFICAS}

1. Wright $T$, Bonett D. Job satisfaction and psychological well-being as nonadditive predictors of workplace turnover. J Nurs Manag. 2007; 33: $141-60$.

2. Shaun S. Satisfacción Laboral. México: Trillas. 1998. pp. 19.

3. Alles A. Comportamiento organizacional: cómo lograr un cambio cultural a través de la Gestión por competencias. Buenos Aires. Granica. 2007. pp. 15-82.

4. Schermerhorn J, Hunt J, Osborn R. Comportamiento Organizacional. México: Limusa Wiley. 2005.

5. Munchinsky P. Psychology applied to work: an introduction to industrial and organizational psychology. Córdoba: Dorsey Press. 2011. pp. 1047-68.

6. Falatah R, Conway E. Linking relational coordination to nurses job satisfaction, affective commitment, and turnover intention in Saudi Arabia. J Nurs Manag. 2018; 27(4): 715-21.

7. Montoya M. Evaluación de la satisfacción laboral y valor percibido por el empleado en la Cámara de Comercio de Santa Rosa de Cabal [Tesis]. Madrid: Universidad Tecnológica de Pereira. Facultad de Ingeniería Industrial; 2014.

8. Neyra Centeno GR, Salinas Valverde FN. Satisfacción laboral de la enfermera o profesional en el Servicio de Emergencia de un hospital nacional [Tesis]. Lima: Universidad Peruana Cayetano Heredia. Facultad de Enfermería; 2017.

9. Pérez Ciordia F. Satisfacción laboral y factores de mejora en profesionales de atención primaria. An Sist Sanit Navar. 2013; 36(2): 253-62.

10. Carballo Chiñas A, Priego Álvarez HR, Ávalos García MI. Clima organizacional y satisfacción laboral en el personal operativo federal 
del programa Caravanas de la Salud en Tabasco, México. Revista UNAM. 2015; 21(59): 43-52.

11. Salinas-Oviedo C, Laguna-Calderón J, Mendoza-Martínez MR. La satisfacción laboral y su papel en la evaluación de la calidad de atención médica-Instituto Nacional de Salud Pública, Mexico. Rev Salud Pública Méx. 1994; 36(1): 22-9.

12. Saturno P, Quintana J, Varo J. Gascón P, Parra P. ¿Qué es la calidad? Tratado de calidad asistencial en atención primaria. Madrid: Dupont Pharma. 1997. pp. 19-45.

13. Carrillo Garcia C, Martinez Roche M, Garcia Maiquez C, Vivo Molina M, Melendreras Montesinos E. Satisfacción laboral del personal de enfermería del quirófano del Hospital Universitario José María Morales Meseguer. Murcia España. RFS Revista Facultad De Salud. 2016; 8(2): 9-15.

14. Fernández San Martín MI, Moinelo Camporro A, Villanueva Guerra A, Andrade Rosa C, Rivera Teijido M, Gómez Ocaña JM, et al. Satisfacción laboral de los profesionales de atención primaria del área 10 del InSalud de Madrid. Rev Esp Salud Pública. 2000; 74(2): 00.

15. Morales Carcelén EM. Nivel de satisfacción laboral del profesional de enfermería en el servicio de centro quirúrgico Hospital Nacional Alberto Sabogal Sologuren, EsSalud, 2016. Lima: Universidad Nacional Mayor de San Marcos. Facultad de Medicina Humana; 2016.

16. Parra Suarez RJ, Arce Vera MF. La satisfacción laboral y su efecto en la satisfacción del cliente, un análisis teórico. INNOVA Research Journal. 2018; 3(8): 157-62.

17. Lovelock C. Administración de servicio. México: Pearson Educación. 2011.

18. Salinas-Oviedo C, Laguna-Calderón J, Mendoza-Martínez MR. La satisfacción laboral y su papel en la evaluación de la calidad de la atención médica. Salud Pública Méx. 1994; 36(1): 22-9.

19. Rodríguez A, Gómez F, De Dios DV. Estudio de la satisfacción laboral en los equipos de atención primaria en un área sanitaria de Asturias. Enferm Global. 2017; 47: 369-83.

20. Pérez-Fonseca M, González de la Cruz P, Castañón-Eugenio I, MoralesHerrera K. Satisfacción laboral del personal de enfermería en una institución de segundo nivel. Rev Técnicas Enferm. 2017; 1(1): 23-9.

\section{Correspondencia}

Carlos W. Contreras Camarena

Dirección: Av. Oscar Benavides 3046. Torre A-403. Lima 01

Teléfono: 912543776

Correo electrónico: ccontrerashndm@hotmail.com

Recibido: 08 de enero de 2020.

Evaluado: 15 de junio de 2020.

Aprobado: 22 de junio de 2020.

(c) La revista. Publicado por Universidad de San Martín de Porres, Perú. (cc) в bajo términos de Licencia Creative Commons Atribución 4.0 Internacional. (http://creativecommons.org/licenses/by/4.0/)

ORCID iDs

Yojani Holguín Morales Chttps://orcid.org/0000-0003-4648-1754

Carlos W. Contreras Camarena i https://orcid.org/0000-0002-7394-995x 